\title{
Note on the Solution of Transport Equation by Tau Method and Walsh Functions
}

\author{
Abdelouahab Kadem ${ }^{1}$ and Adem Kilicman ${ }^{2}$ \\ ${ }^{1}$ L.M.F.N Mathematics Department, University of Setif, Setif 19000, Algeria \\ ${ }^{2}$ Department of Mathematics and Institute for Mathematical Research, University Putra Malaysia (UPM), \\ Serdang, Selangor 43400, Malaysia
}

Correspondence should be addressed to Adem Kilicman, akilicman@putra.upm.edu.my

Received 9 November 2010; Accepted 27 December 2010

Academic Editor: Yoshikazu Giga

Copyright (C 2010 A. Kadem and A. Kilicman. This is an open access article distributed under the Creative Commons Attribution License, which permits unrestricted use, distribution, and reproduction in any medium, provided the original work is properly cited.

\begin{abstract}
We consider the combined Walsh function for the three-dimensional case. A method for the solution of the neutron transport equation in three-dimensional case by using the Walsh function, Chebyshev polynomials, and the Legendre polynomials are considered. We also present Tau method, and it was proved that it is a good approximate to exact solutions. This method is based on expansion of the angular flux in a truncated series of Walsh function in the angular variable. The main characteristic of this technique is that it reduces the problems to those of solving a system of algebraic equations; thus, it is greatly simplifying the problem.
\end{abstract}

\section{Introduction}

The Walsh functions have many properties similar to those of the trigonometric functions. For example, they form a complete, total collection of functions with respect to the space of square Lebesgue integrable functions. However, they are simpler in structure to the trigonometric functions because they take only the values 1 and -1 . They may be expressed as linear combinations of the Haar functions [1], so many proofs about the Haar functions carry over to the Walsh system easily. Moreover, the Walsh functions are Haar wavelet packets. For a good account of the properties of the Haar wavelets and other wavelets, see [2]. We use the ordering of the Walsh functions due to Paley $[3,4]$. Any function $f \in L^{2}[0,1]$ can be expanded as a series of Walsh functions

$$
f(x)=\sum_{i=0}^{\infty} c_{i} W_{i}(x), \quad \text { where } c_{i}=\int_{0}^{1} f(x) W_{i}(x)
$$


In [5], Fine discovered an important property of the Walsh Fourier series: the $m=2^{n}$ th partial sum of the Walsh series of a function $f$ is piecewise constant, equal to the $L^{1}$ mean of $f$, on each subinterval $[(i-1) / m, i / m]$. For this reason, Walsh series in applications are always truncated to $m=2^{n}$ terms. In this case, the coefficients $c_{i}$ of the Walsh (-Fourier) series are given by

$$
c_{i}=\sum_{j=0}^{m-1} \frac{1}{m} W_{i j} f_{j},
$$

where $f_{j}$ is the average value of the function $f(x)$ in the $j$ th interval of width $1 / m$ in the interval $(0,1)$, and $W_{i j}$ is the value of the $i$ th Walsh function in the $j$ th subinterval. The order $m$ Walsh matrix, $\mathcal{W}_{m}$, has elements $W_{i j}$.

Let $f(x)$ have a Walsh series with coefficients $c_{i}$ and its integral from 0 to $x$ have a Walsh series with coefficients $b_{i}: \int_{0}^{x} f(t) d t=\sum_{i=0}^{\infty} b_{i} W_{i}(x)$. If we truncate to $m=2^{n}$ terms and use the obvious vector notation, then integration is performed by matrix multiplication $\mathbf{b}=P_{m}^{T} \mathbf{c}$, where

$$
P_{m}^{T}=\left[\begin{array}{cc}
P_{m / 2} & \frac{1}{2 m} I_{m / 2} \\
-\frac{1}{2 m} I_{m / 2} & O_{m / 2}
\end{array}\right], \quad P_{2}^{T}=\left[\begin{array}{cc}
\frac{1}{2} & \frac{1}{4} \\
-\frac{1}{4} & 0
\end{array}\right]
$$

and $I_{m}$ is the unit matrix, $O_{m}$ is the zero matrix (of order $m$ ), see [6].

\section{The Three-Dimensional Spectral Solution}

In the literature there several works on driving a suitable model for the transport equation in 2 and 3-dimensional case as well as in cylindrical domain, for example, see [7], and by using the eigenvalue error estimates for two-dimensional neutron transport, see [8], by applying the finite element method in an infinite cylindrical domain, see [9], similarly by using Chebyshev spectral- $S_{N}$ method, see [10], and the discrete ordinates in the infinite cylindrical domain, see [11].

In this paper, we consider combined Walsh function with the Sumudu transform in order to extend the transport problem for the three-dimensional case by following the similar method that was proposed in [7]. This method is based on expansion of the angular flux in a truncated series of Walsh function in the angular variable. By replacing this development in the transport equation, this will result a first-order linear differential system. First of all we consider the three-dimensional linear, steady state, transport equation which is given by

$$
\begin{gathered}
\mu \frac{\partial}{\partial x} \Psi(\mathbf{x}, \mu, \theta)+\sqrt{1-\mu^{2}}\left(\cos \theta \frac{\partial}{\partial y} \Psi(\mathbf{x}, \mu, \theta)+\sin \theta \frac{\partial}{\partial z} \Psi(\mathbf{x}, \mu, \theta)\right)+\sigma_{t} \Psi(\mathbf{x}, \mu, \theta) \\
=\int_{-1}^{1} \int_{0}^{2 \pi} \sigma_{s}\left(\mu^{\prime}, \theta^{\prime} \longrightarrow \mu, \theta\right) \Psi\left(\mathbf{x}, \mu^{\prime}, \theta^{\prime}\right) d \theta^{\prime} d \mu^{\prime}+S(\mathbf{x}, \mu, \theta),
\end{gathered}
$$


where we assume that the spatial variable $\mathbf{x}:=(x, y, z)$ varies in the cubic domain $\Omega:=$ $\{(x, y, z):-1 \leq x, y, z \leq 1\}$, and $\Psi(\mathbf{x}, \mu, \theta):=\Psi(x, y, z, \mu, \theta)$ is the angular flux in the direction defined by $\mu \in[-1,1]$ and $\theta \in[0,2 \pi]$. $\sigma_{t}$ and $\sigma_{\mathrm{s}}$ denote the total and the differential cross section, respectively, $\sigma_{s}\left(\mu^{\prime}, \phi^{\prime} \rightarrow \mu, \phi\right)$ describes the scattering from an assumed pre-collision angular coordinates $\left(\mu^{\prime}, \theta^{\prime}\right)$ to a postcollision coordinates $(\mu, \theta)$ and $S$ is the source term. See [12] for further details.

Note that, in the case of one-speed neutron transport equation; taking the angular variable in a disc, this problem will corresponds to a three dimensional case with all functions being constant in the azimuthal direction of the $z$ variable. In this way the actual spatial domain may be assumed to be a cylinder with the cross-section $\Omega$ and the axial symmetry in $z$. Then $D$ will correspond to the projection of the points on the unit sphere (the "speed") onto the unit disc (which coincides with $D$ ). See [13] for the details.

Given the functions $f_{1}(y, z, \mu, \phi), f_{2}(x, z, \mu, \phi)$, and $f_{3}(x, y, \mu, \phi)$ describing the incident flux, we seek for a solution of (2.1) subject to the following boundary conditions.

For the boundary terms in $x$, for $0 \leq \theta \leq 2 \pi$, let

$$
\Psi(x= \pm 1, y, z, \mu, \theta)= \begin{cases}f_{1}(y, z, \mu, \theta), & x=-1,0<\mu \leq 1 \\ 0, & x=1,-1 \leq \mu<0 .\end{cases}
$$

For the boundary terms in $y$ and for $-1 \leq \mu<1$,

$$
\Psi(x, y= \pm 1, z, \mu, \theta)= \begin{cases}f_{2}(x, z, \mu, \theta), & y=-1,0<\cos \theta \leq 1 \\ 0, & y=1,-1 \leq \cos \theta<0\end{cases}
$$

Finally, for the boundary terms in $z$, for $-1 \leq \mu<1$,

$$
\Psi(x, y, z= \pm 1, \mu, \theta)= \begin{cases}f_{3}(x, y, \mu, \theta), & z=-1,0 \leq \theta<\pi \\ 0, & z=1, \pi<\theta \leq 2 \pi\end{cases}
$$

Theorem 2.1. Consider the integrodifferential equation (2.1) under the boundary conditions (2.2), (2.3) and (2.4), then the function $\Psi(x, y, z, \mu, \theta)$ satisfy the following first-order linear differential equation system for the spatial component $\Psi_{i, j}(x, \mu, \theta)$

$$
\begin{aligned}
& \mu \frac{\partial \Psi_{i, j}}{\partial x}(x, \mu, \theta)+\sigma_{t} \Psi_{i, j}(x, \mu, \theta) \\
& \quad=G_{i, j}(x ; \mu, \theta) \iint_{-1}^{1} \sigma_{s}\left(\mu^{\prime}, \theta^{\prime} \longrightarrow \mu, \theta\right) \Psi_{i, j}\left(x, \mu^{\prime}, \theta^{\prime}\right) d \theta^{\prime} d \mu^{\prime},
\end{aligned}
$$

with the boundary conditions

$$
\Psi_{i, j}(-1, \mu, \eta)=f_{1}^{i, j}(\mu, \theta)
$$


where

$$
\begin{gathered}
f_{1}^{i, j}(\mu, \theta)=\frac{4}{\pi^{2}} \iint_{-1}^{1} \frac{T_{i}(y) R_{j}(z)}{\sqrt{\left(1-y^{2}\right)\left(1-z^{2}\right)}} f_{1}(y, z, \mu, \theta) d z d y, \\
\Psi_{i, j}(1,-\mu, \theta)=0, \\
G_{i, j}(x ; \mu, \theta)=S_{i, j}(x, \mu, \theta)-\sqrt{1-\mu^{2}}\left[\cos \theta \sum_{k=i+1}^{I} A_{i}^{k} \Psi_{k, j}(x, \mu, \theta)+\sin \theta \sum_{l=j+1}^{J} B_{j}^{l} \Psi_{i, l}(x, \mu, \theta)\right],
\end{gathered}
$$

with

$$
\begin{gathered}
S_{i, j}(x, \mu, \theta)=\frac{4}{\pi^{2}} \iint_{-1}^{1} \frac{T_{i}(y) R_{j}(z)}{\sqrt{\left(1-y^{2}\right)\left(1-z^{2}\right)}} S(\mathbf{x}, \mu, \theta) d z d y, \\
A_{i}^{k}=\frac{2}{\pi} \int_{-1}^{1} \frac{d}{d y}\left(T_{k}(y)\right) \frac{T_{i}(y)}{\sqrt{1-y^{2}}} d y, \\
B_{j}^{l}=\frac{2}{\pi} \int_{-1}^{1} \frac{d}{d y}\left(R_{l}(y)\right) \frac{R_{j}(z)}{\sqrt{1-z^{2}}} d z .
\end{gathered}
$$

Proof. Expanding the angular flux $\Psi(x, y, z, \mu, \phi)$ in a truncated series of Chebyshev polynomials $T_{i}(y)$ and $R_{j}(z)$ leads to

$$
\Psi(x, y, z, \mu, \theta)=\sum_{i=0}^{I} \sum_{j=0}^{J} \Psi_{i, j}(x, \mu, \theta) T_{i}(y) R_{j}(z)
$$

We insert $\Psi(x, y, z, \mu, \theta)$ given by (2.9) into the boundary condition in (2.3), for $y= \pm 1$. Multiplying the resulting expressions by $R_{j}(z) / \sqrt{1-z^{2}}$ and integrating over $z$, we get the components $\Psi_{0, j}(x, \mu, \theta)$ for $j=0, \ldots, J$,

$$
\begin{gathered}
\Psi_{0, j}(x, \mu, \theta)=f_{2}^{j}(x, \mu, \theta)-\sum_{i=1}^{I}(-1)^{j} \Psi_{i, j}(x, \mu, \theta), \quad 0<\cos \theta \leq 1, \\
\Psi_{0, j}(x, \mu, \theta)=-\sum_{i=1}^{I} \Psi_{i, j}(x, \mu, \theta), \quad-1 \leq \cos \theta<0 .
\end{gathered}
$$


Similarly, we substitute $\Psi(x, y, z, \mu, \theta)$ from (2.9) into the boundary conditions for $z= \pm 1$, multiply the resulting expression by $T_{i}(y) / \sqrt{1-y^{2}}, i=0, \ldots, I$ and integrating over $y$, to define the components $\Psi_{i, 0}(x, \mu, \theta)$. For $-1 \leq x \leq 1,-1<\mu<1$,

$$
\begin{gathered}
\Psi_{i, 0}(x, \mu, \theta)=f_{3}^{i}(x, \mu, \theta)-\sum_{j=1}^{J}(-1)^{j} \Psi_{i, j}(x, \mu, \theta), \quad 0 \leq \theta<\pi, \\
\Psi_{i, 0}(x, \mu, \theta)=-\sum_{j=1}^{J} \Psi_{i, j}(x, \mu, \theta), \quad \pi<\theta \leq 2 \pi,
\end{gathered}
$$

where

$$
\begin{aligned}
& f_{2}^{\beta}(x, \mu, \theta)=\frac{2-\delta_{0, j}}{\pi} \int_{-1}^{1} f_{2}(x, z, \mu, \theta) \frac{R_{j}(z)}{\sqrt{1-z^{2}}} d z, \\
& f_{3}^{i}(x, \mu, \theta)=\frac{2-\delta_{i, 0}}{\pi} \int_{-1}^{1} f_{3}(x, y, \mu, \theta) \frac{T_{i}(y)}{\sqrt{1-y^{2}}} d y .
\end{aligned}
$$

To determine the components $\Psi_{i, j}(x, \mu, \theta), i=1, \ldots, I$, and $j=1, \ldots, J$ we substitute $\Psi(x, \mu, \theta)$, from (2.3) into (2.1) and the boundary conditions for $x= \pm 1$. Multiplying the resulting expressions by $\left(T_{i}(y) / \sqrt{1-y^{2}}\right) \times\left(R_{j}(z) / \sqrt{1-z^{2}}\right)$, and integrating over $y$ and $z$ we obtain $I \times J$ one-dimensional transport problems, namely,

$$
\mu \frac{\partial \Psi_{i, j}}{\partial x}(x, \mu, \theta)+\sigma_{t} \Psi_{i, j}(x, \mu, \theta)=G_{i, j}(x ; \mu, \theta) \iint_{-1}^{1} \sigma_{s}\left(\mu^{\prime}, \theta^{\prime} \longrightarrow \mu, \theta\right) \Psi_{i, j}\left(x, \mu^{\prime}, \theta^{\prime}\right) d \theta^{\prime} d \mu^{\prime},
$$

with the boundary conditions

$$
\Psi_{i, j}(-1, \mu, \eta)=f_{1}^{i, j}(\mu, \theta)
$$

where

$$
\begin{gathered}
f_{1}^{i, j}(\mu, \theta)=\frac{4}{\pi^{2}} \iint_{-1}^{1} \frac{T_{i}(y) R_{j}(z)}{\sqrt{\left(1-y^{2}\right)\left(1-z^{2}\right)}} f_{1}(y, z, \mu, \theta) d z d y, \\
\Psi_{i, j}(1,-\mu, \theta)=0
\end{gathered}
$$


for $0<\mu \leq 1$, and $0 \leq \theta \leq 2 \pi$. Finally,

$$
G_{i, j}(x ; \mu, \theta)=S_{i, j}(x, \mu, \theta)-\sqrt{1-\mu^{2}}\left[\cos \theta \sum_{k=i+1}^{I} A_{i}^{k} \Psi_{k, j}(x, \mu, \theta)+\sin \theta \sum_{l=j+1}^{J} B_{j}^{l} \Psi_{i, l}(x, \mu, \theta)\right],
$$

with

$$
\begin{aligned}
S_{i, j}(x, \mu, \theta) & =\frac{4}{\pi^{2}} \iint_{-1}^{1} \frac{T_{i}(y) R_{j}(z)}{\sqrt{\left(1-y^{2}\right)\left(1-z^{2}\right)}} S(\mathbf{x}, \mu, \theta) d z d y, \\
A_{i}^{k} & =\frac{2}{\pi} \int_{-1}^{1} \frac{d}{d y}\left(T_{k}(y)\right) \frac{T_{i}(y)}{\sqrt{1-y^{2}}} d y, \\
B_{j}^{l} & =\frac{2}{\pi} \int_{-1}^{1} \frac{d}{d y}\left(R_{l}(y)\right) \frac{R_{j}(z)}{\sqrt{1-z^{2}}} d z .
\end{aligned}
$$

Now, starting from the solution of the problem given by (2.13)-(2.17) for $\Psi_{I, J}(x, \mu, \theta)$, we then solve the problems for the other components, in the decreasing order in $i$ and $j$. Recall that $\sum_{i=I+1}^{I} \cdots=\sum_{j=J+1}^{J} \equiv 0$. Hence, solving $I \times J$ one-dimensional problems, the angular flux $\Psi(\mathbf{x}, \mu, \theta)$ is now completely determined through (2.9).

Remark 2.2. If we deal with different type of boundary conditions, then we consider the first components $\Psi_{i, 0}(x, \mu, \theta)$ and $\Psi_{0, j}(x, \mu, \theta)$ for $i=1, \ldots, I$ and $j=1, \ldots, J$ will satisfy onedimensional transport problems subject to the same of boundary conditions of the original problem in the variable $x$.

\section{Analysis}

Now, we solve the first-order linear differential equation system with isotropic scattering, that is, $\sigma_{s}\left(\mu^{\prime}, \phi^{\prime} \rightarrow \mu, \phi\right) \equiv \sigma_{s}=$ constant. Assuming isotropic scattering, the equation (2.13) is written as

$$
\mu \frac{\partial \Psi_{i, j}}{\partial x}(x, \mu, \theta)+\sigma_{t} \Psi_{i, j}(x, \mu, \theta)=G_{i, j}(x ; \mu, \theta) \sigma \int_{-1}^{1} \int_{0}^{2 \pi} \Psi_{i, j}\left(x, \mu^{\prime}, \theta^{\prime}\right) d \theta^{\prime} d \mu^{\prime},
$$

for $\mathbf{x} \in \Omega:=\{(x, y): 0 \leq x \leq 1,-1 \leq y \leq 1\}, \mu \in[-1,1]$, and $\theta \in[0,2 \pi]$.

Then, we have the following theorem that is subject to the boundary conditions (2.14). 
Theorem 3.1. Consider the integrodifferential equation (3.1) under the boundary conditions (2.14), then the function $\Psi_{i, j}(x, \mu, \theta)$ satisfy the following linear system of algebraic equations:

$$
\begin{array}{r}
\sum_{n=0}^{N} D_{n, m} p \bar{\beta}_{n, i, j}(p, \theta)-\sigma_{s} \sum_{n=0}^{N} p \bar{\alpha}_{n, i, j}(p, \theta)+\sigma_{t} \bar{\alpha}_{n, i, j}(p, \theta) \\
=\int_{-1}^{1} \bar{G}_{i, j}(x, \mu, \theta) W_{n}^{e}(\mu) d \mu+\sum_{n=0}^{N} D_{n, m} \beta_{n, i, j}(0, \theta), \\
\sum_{n=0}^{N} D_{n, m} p \bar{\alpha}_{n, i, j}(p, \theta)-\sigma_{s} \sum_{n=0}^{N} p \bar{\beta}_{n, k}(p, \theta)+\sigma_{t} \bar{\beta}_{n, i, j}(p, \theta) \\
=\int_{-1}^{1} \bar{G}_{i, j}(x, \mu, \theta) W_{n}^{o}(\mu) d \mu+\sum_{n=0}^{N} D_{n, m} \bar{\alpha}_{n, i, j}(0, \theta) .
\end{array}
$$

Proof. For this problem we expand the angular flux in terms of the Walsh function in the angular variable with its domain extended into the interval $[-1,1]$. To end this, the Walsh function $W_{n}(\mu)$ are extended in an even and odd fashion as follows, see [14]:

$$
\begin{gathered}
W_{n}^{e}(\mu)= \begin{cases}W_{n}(\mu) & \text { if } \mu \geq 0, \\
W_{n}(-\mu) & \text { if } \mu<0,\end{cases} \\
W_{n}^{o}(\mu)= \begin{cases}W_{n}(\mu) & \text { if } \mu \geq 0, \\
-W_{n}(-\mu) & \text { if } \mu<0,\end{cases}
\end{gathered}
$$

for $n=0,1, \ldots, N$. The important feature of this procedure relies on the fact that a function $f(\mu)$ is defined in the interval $[-1,1]$ it can be expanded in terms of these extended functions in the manner:

$$
f(\mu)=\sum_{n=0}^{\infty}\left[a_{n} W_{n}^{e}(\mu)+b_{n} W_{n}^{o}(\mu)\right]
$$

where the coefficients $a_{n}$ and $b_{n}$ are determined as

$$
\begin{aligned}
& a_{n}=\frac{1}{2} \int_{-1}^{1} f(\mu) W_{n}^{e}(\mu) d \mu, \\
& b_{n}=\frac{1}{2} \int_{-1}^{1} f(\mu) W_{n}^{o}(\mu) d \mu .
\end{aligned}
$$


So, in order to use the Walsh function for the solution of the problem (3.1), the angular flux is approximated by the truncated expansion:

$$
\Psi_{i, j}(x, \mu, \theta)=\sum_{n=0}^{N}\left[\alpha_{n, i, j}(x, \theta) W_{n}^{e}(\mu)+\beta_{n, i, j}(x, \theta) W_{n}^{o}(\mu)\right]
$$

Inserting this expansion into the linear transport (3.1), it turns out

$$
\begin{aligned}
\sum_{n=0}^{N}\left[\left\{\mu \frac{\partial \alpha_{n, i, j}}{\partial x}(x, \theta)+\sigma_{t} \alpha_{n, i, j}(x, \theta)\right\} W_{n}^{e}(\mu)+\left\{\mu \frac{\partial \beta_{n, i, j}}{\partial x}(x, \theta)+\sigma_{t} \beta_{n, i, j}(x, \theta)\right\} W_{n}^{o}(\mu)\right] \\
=\sum_{n=0}^{N} \sigma_{s}\left[\int_{-1}^{1} \int_{0}^{2 \pi} \alpha_{n, i, j}\left(x, \theta^{\prime}\right) W_{n}^{e}\left(\mu^{\prime}\right) d \theta^{\prime} d \mu^{\prime}+\int_{-1}^{1} \int_{0}^{2 \pi} \alpha_{n, i, j}\left(x, \theta^{\prime}\right) W_{n}^{o}\left(\mu^{\prime}\right) d \theta^{\prime} d \mu^{\prime}\right] \\
\quad+G_{i, j}(x, \mu, \theta) .
\end{aligned}
$$

Multiplying (3.7) by $W_{m}^{e}, m=0, \ldots, N$ and integrating over the interval $[-1,1]$, results:

$$
\begin{aligned}
\sum_{n=0}^{N}\left[\frac{\partial \beta_{n, i, j}}{\partial x}(x, \theta) \int_{-1}^{1} \mu W_{n}^{o}(\mu) W_{n}^{e}(\mu) d \mu\right]+\sigma_{t} \alpha_{n, i, j}(x, \theta) \int_{-1}^{1} W_{n}^{e}(\mu) W_{m}^{e}(\mu) d \mu \\
\quad=\sum_{n=0}^{N} \sigma_{s}\left[\int_{0}^{2 \pi} \alpha_{n, i, j}\left(x, \theta^{\prime}\right) d \theta^{\prime} \int_{-1}^{1} W_{n}^{o}\left(\mu^{\prime}\right) W_{n}^{o}\left(\mu^{\prime}\right) d \mu^{\prime}\right]+\int_{-1}^{1} G_{i, j}(x, \mu, \theta) W_{n}^{e}(\mu) d \mu .
\end{aligned}
$$

Similarly, multiplying (3.7) by $W_{m}^{0}, m=0, \ldots, N$ and integrating yields:

$$
\begin{aligned}
& \sum_{n=0}^{N}\left[\frac{\partial \alpha_{n, i, j}}{\partial x}(x, \theta) \int_{-1}^{1} \mu W_{n}^{o}(\mu) W_{n}^{e}(\mu) d \mu+\sigma_{t} \beta_{n, i, j}(x, \theta) \int_{-1}^{1} W_{n}^{0}(\mu) W_{m}^{0}(\mu) d \mu\right] \\
& \quad=\sum_{n=0}^{N} \sigma_{s}\left[\int_{0}^{2 \pi} \beta_{n, i, j}\left(x, \theta^{\prime}\right) d \theta^{\prime} \int_{-1}^{1} W_{n}^{o}\left(\mu^{\prime}\right) W_{n}^{o}\left(\mu^{\prime}\right) d \mu^{\prime}\right]+\int_{-1}^{1} G_{i, j}(x, \mu, \theta) W_{n}^{0}(\mu) d \mu .
\end{aligned}
$$

The integrals appearing in (3.8) and (3.9) are known and are given [14] as

$$
D_{n, m}=\frac{1}{2} \int_{-1}^{1} \mu W_{n}^{o}(\mu) W_{m}^{e}(\mu) d \mu=\int_{0}^{1} \mu W_{(n+m) \bmod 2}(\mu)
$$

or

$$
D_{n, m}= \begin{cases}\frac{1}{2} & \text { if } n=m \\ -2^{-(k+2),}, & \text { if }(n+m) \bmod 2=2^{k} k \text { natural } \\ 0 & \text { at another case }\end{cases}
$$


where the notation $(n+m) \bmod 2$ denotes the $\bmod 2$ sum of the binary digits $n$ and $m$ [15]

$$
\begin{array}{r}
\sum_{n=0}^{N} D_{n, m} p \bar{\beta}_{n, i, j}(p, \theta)-\sigma_{s} \sum_{n=0}^{N} p \bar{\alpha}_{n, i, j}(p, \theta)+\sigma_{t} \bar{\alpha}_{n, i, j}(p, \theta) \\
=\int_{-1}^{1} \bar{G}_{i, j}(x, \mu, \theta) W_{n}^{e}(\mu) d \mu+\sum_{n=0}^{N} D_{n, m} \beta_{n, i, j}(0, \theta), \\
\sum_{n=0}^{N} D_{n, m} p \bar{\alpha}_{n, i, j}(p, \theta)-\sigma_{s} \sum_{n=0}^{N} p \bar{\beta}_{n, k}(p, \theta)+\sigma_{t} \bar{\beta}_{n, i, j}(p, \theta) \\
=\int_{-1}^{1} \bar{G}_{i, j}(x, \mu, \theta) W_{n}^{o}(\mu) d \mu+\sum_{n=0}^{N} D_{n, m} \bar{\alpha}_{n, i, j}(0, \theta) .
\end{array}
$$

\section{Operational Tau Method and Converting Transport Equation}

The operational approach to the Tau method proposed by [16] describes converting of a given integral, integrodifferential equation or system of these equations to a system of linear algebraic equations based on three simple matrices:

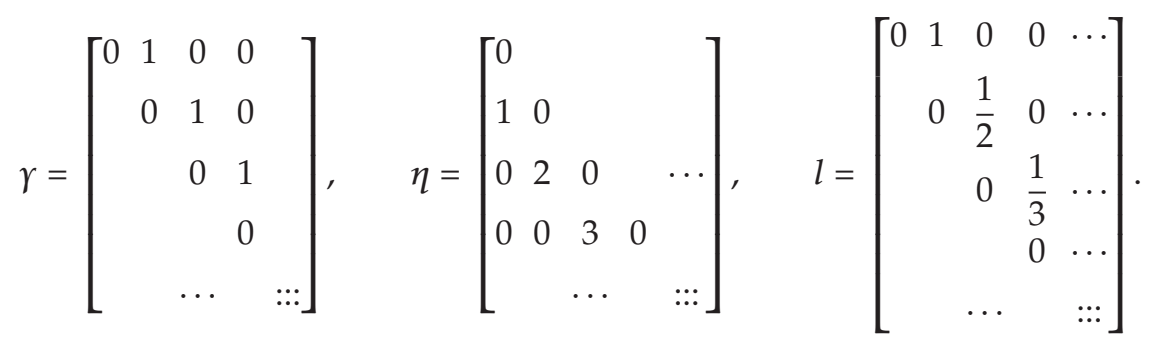

We recall the following properties [17].

Lemma 4.1. Let $u_{n}(x)$ be a polynomial as

$$
u_{n}(x)=\sum_{i=0}^{n} a_{i} x^{i}=\sum_{i=0}^{\infty} a_{i} x^{i}=\overrightarrow{a_{n}} X
$$

then we have

(i) $\left(d^{r} / d x^{r}\right) u_{n}(x)=\overrightarrow{a_{n}} \eta^{r} X, r=1,2,3, \ldots$,

(ii) $x^{r} u_{n}(x)=\overrightarrow{a_{n}} \gamma^{r} X, r=1,2,3, \ldots$,

(iii) $\int_{a}^{x} u_{n}(x) d x=\left.\overrightarrow{a_{n}} l X\right|_{a} ^{x}=\overrightarrow{a_{n}} l X-\overrightarrow{a_{n}} l A$,

where $\vec{a}_{n}=\left[a_{0}, a_{1}, \ldots, a_{n}, 0,0, \ldots\right]$ and $A=\left[1, a, a^{2}, \ldots, a^{n}, \ldots\right]^{T}$. 
Now we solve (3.12) using the Tau method, for this we consider this equation because is equivalent

$$
\mu \frac{\partial \Psi_{i, j}}{\partial x}(x, \mu, \theta)+\sigma_{t} \Psi_{i, j}(x, \mu, \theta)=G_{i, j}(x ; \mu, \theta)+\int_{-1}^{1} \int_{0}^{2 \pi} \sigma_{s}\left(\mu^{\prime}, \theta^{\prime}\right) \Psi_{i, j}\left(x, \mu^{\prime}, \theta^{\prime}\right) d \theta^{\prime} d \mu^{\prime}
$$

for $\mathbf{x} \in \Omega:=\{(x, y): 0 \leq x \leq 1,-1 \leq y \leq 1\}, \mu \in[-1,1]$, and $\theta \in[0,2 \pi]$ subject to the following boundary conditions (2.14).

In order to convert (5.1) to a system of linear algebraic equations we define the linear differential operator $D$ of order $\zeta$ with polynomial coefficients defined by

$$
D:=\sum_{r=0}^{\zeta} p_{r}(x) \frac{\partial^{r}}{\partial x^{r}}
$$

we will write for

$$
p_{r}(x):=\sum_{k=0}^{\alpha_{r}} p_{r k} x^{k}
$$

where $\alpha_{r}$ is the degree of $p_{r}(x)$ and $p_{r}=\left(p_{r 0, \ldots,} p_{r \alpha_{r}}, 0,0, \ldots\right), \underline{\Upsilon}=\left(1, \mu, \mu^{2}, \ldots\right)^{T}$. We notice that $\mu$ is the independent variable and will be defined in a finite interval.

\subsection{Matrix Representation for the Different Parts}

Let $V=\left\{v_{0}(\mu), v_{1}(\mu), \ldots\right\}$ be a polynomial basis given by $\underline{V}:=V \underline{\Upsilon}$, where $V$ is nonsingular lower triangular matrix and degree $\left(v_{i}(\mu)\right) \leq i$, for $i=0,1,2, \ldots$, Also for any matrix $P$, $P_{v}=V P V^{-1}$.

Matrix Representation for $D \Psi_{i, j}(x, \mu, \theta)$

Theorem 4.2 (see [16]). For any linear differential operator $D$ defined by (5.2) and any series

$$
\begin{gathered}
\Psi_{i, j}(x, \mu, \theta): \underline{\underline{a} V}, \\
\underline{\underline{a}}:=\left(\Psi_{0, j}\left(x, \theta^{\prime}\right), \Psi_{1, j}\left(x, \theta^{\prime}\right), \Psi_{2, j}\left(x, \theta^{\prime}\right), \ldots, \Psi_{i, 0}\left(x, \theta^{\prime}\right), \Psi_{i, 1}\left(x, \theta^{\prime}\right), \Psi_{i, 2}\left(x, \theta^{\prime}\right), \ldots,\right),
\end{gathered}
$$

we have

$$
D \Psi_{i, j}(x, \mu, \theta)=\underline{\underline{a}} \Xi_{v} \underline{V}
$$


where

$$
\Xi_{v}=V \sum_{i=0}^{\zeta} \eta^{i} p_{i}(\gamma) V^{-1}
$$

Matrix Representation for the Integral Form

Let us assume that

$$
\begin{gathered}
\sigma_{s}\left(\mu^{\prime}, \theta^{\prime}\right)=\sum_{j=0}^{n} \sigma_{s}\left(\theta^{\prime}\right) v_{j}\left(\mu^{\prime}\right), \\
\Psi_{i, j}\left(x, \mu^{\prime}, \theta^{\prime}\right)=\sum_{i, j=0}^{n} \Psi_{i, j}\left(x, \theta^{\prime}\right) v_{i}\left(\mu^{\prime}\right)=\underline{\underline{a} V},
\end{gathered}
$$

then we can write

$$
\begin{aligned}
\int_{-1}^{1} \int_{0}^{2 \pi} \sigma_{S}\left(\mu^{\prime}, \theta^{\prime}\right) \Psi_{i, j}\left(x, \mu^{\prime}, \theta^{\prime}\right) d \theta^{\prime} d \mu^{\prime} & =\sum_{i, j=0}^{n} \int_{0}^{2 \pi} \sigma_{s}\left(\theta^{\prime}\right) \Psi_{i, j}\left(x, \mu^{\prime}, \theta^{\prime}\right) d \theta^{\prime} \int_{-1}^{1} v_{j}\left(\mu^{\prime}\right) v_{i}\left(\mu^{\prime}\right) d \mu^{\prime} \\
& =\underline{\underline{a} K V},
\end{aligned}
$$

where

$$
K=\left[\begin{array}{cccccc}
\sum_{j=0}^{n} k_{0, j} \varpi_{j 0} & \cdots & \sum_{i, j=0}^{n} k_{0, j} \varpi_{i j} & 0 & 0 & \cdots \\
\ldots & \cdots & \cdots & \cdots & \ldots & \cdots \\
\sum_{j=0}^{n} k_{0, j} \varpi_{i j} & \cdots & \sum_{i, j=0}^{n} k_{0, j} \varpi_{i j} & 0 & 0 & \cdots \\
\cdots & \ldots & \cdots & \ldots & \ldots & \cdots
\end{array}\right],
$$

with $k_{i, j}=\int_{0}^{2 \pi} \sigma_{s}\left(\theta^{\prime}\right), \Psi_{i, j}\left(x, \mu^{\prime}, \theta^{\prime}\right) d \theta^{\prime}$, and $\varpi_{i j}=\int_{-1}^{1} v_{i}\left(\mu^{\prime}\right) v_{j}\left(\mu^{\prime}\right) d \mu^{\prime}$ for $i, j=0,1, \ldots, n$.

Matrix Representation for the Boundary Conditions

$$
\begin{aligned}
\Psi_{i, j}\left(x, \mu^{\prime}, \theta^{\prime}\right) & =\sum_{i=0}^{\infty} \sum_{k=0}^{\zeta} \Psi_{j k}\left(x, \theta^{\prime}\right) v_{j}\left(\mu^{\prime}\right) \\
& =\underline{a} \operatorname{Col}\left[\Psi_{0, j}\left(x, \theta^{\prime}\right), \Psi_{1, j}\left(x, \theta^{\prime}\right), \ldots, \Psi_{i, 0}\left(x, \theta^{\prime}\right), \Psi_{i, 1}\left(x, \theta^{\prime}\right), \ldots,\right]=\underline{a} B_{j},
\end{aligned}
$$


where $j=1, \ldots, \zeta$. It follows from (5.4) and (5.5) that

$$
\begin{aligned}
\mu \frac{\partial \Psi_{i, j}}{\partial x}(x, \mu, \theta)+\sigma_{t} \Psi_{i, j}(x, \mu, \theta) & =\int_{-1}^{1} \int_{0}^{2 \pi} \sigma_{s}\left(\mu^{\prime}, \theta^{\prime}\right) \Psi_{i, j}\left(x, \mu^{\prime}, \theta^{\prime}\right) d \theta^{\prime} d \mu^{\prime} \\
& =\frac{1}{\mu}\left[\underline{\underline{a} K V}-\underline{\underline{a}} \Xi_{v} \underline{V}\right] .
\end{aligned}
$$

Let $G_{i, j}(x ; \mu, \theta)=\sum_{d=0}^{n} G_{i, j d}(x ; \theta) v_{d}(\mu)=\underline{G_{i, j}}(x ; \theta) \underline{V}$ with $G_{i, j}=\left(G_{i, j 0}, \ldots, G_{i, j n}, 0,0, \ldots\right)$.

\section{Error Estimation}

Consider now the discrete ordinates $\left(S_{N}\right)$ approximation of the equation (2.13) for $m=$ $1, \ldots, M$,

$$
\mu_{m} \frac{\partial \Psi_{\alpha, \beta, N}}{\partial x}\left(x, \mu_{m}, \theta\right)+\sigma_{t} \Psi_{\alpha, \beta, N}\left(x, \mu_{m}, \theta\right)=\sum_{n=1}^{M} \omega_{n} \Psi_{\alpha, \beta, N}\left(x, \mu_{m}, \theta\right)+G_{\alpha, \beta}\left(x, \mu_{m}, \theta\right) .
$$

In this part, we evaluate an error estimator for the approximate solution of (2.13), we suppose that equations (2.13) and (5.1) have the same boundary conditions. Let us call

$$
\epsilon_{m}(x, \mu, \theta)=\Psi_{i, j}\left(x, \mu_{m}, \theta\right)-\Psi_{i, j, m}(x, \mu, \theta)
$$

this error function of the approximate solution $\Psi_{i, j, m}$ to $\Psi_{i, j}$ where $\Psi_{i, j}$ is the exact solution of (2.13). Hence, $\Psi_{i, j}\left(x, \mu_{m}, \theta\right)$ satisfies the following problem:

$$
\mu_{m} \frac{\partial \Psi_{\alpha, \beta, N}}{\partial x}\left(x, \mu_{m}, \theta\right)+\sigma_{t} \Psi_{\alpha, \beta, N}\left(x, \mu_{m}, \theta\right)=\sum_{n=1}^{M} \omega_{n} \Psi_{\alpha, \beta, N}\left(x, \mu_{m}, \theta\right)+G_{\alpha, \beta}\left(x, \mu_{m}, \theta\right)+H_{N}\left(\mu_{m}\right)
$$

We can evaluate the perturbation term $H_{N}\left(\mu_{m}\right)$ by substituting the computed solution into the equation

$$
H_{N}\left(\mu_{m}\right)=\mu_{m} \frac{\partial \Psi_{\alpha, \beta, N}}{\partial x}(x, \mu, \theta)+\sigma_{t} \Psi_{\alpha, \beta, N}(x, \mu, \theta)-\sum_{n=1}^{M} \omega_{n} \Psi_{\alpha, \beta, N}\left(x, \mu_{m}, \theta\right)-G_{\alpha, \beta}\left(x, \mu_{m}, \theta\right)
$$

after doing some algebraic manipulations, the error functions $\epsilon_{m}(\mu)$ satisfies the problem

$$
H_{N}\left(\mu_{m}\right)=\mu_{m} \frac{\partial \epsilon_{m}(x, \mu, \theta)}{\partial x}+\sigma_{t} \epsilon_{m}(x, \mu, \theta)-\sum_{n=1}^{M} \omega_{n} \epsilon_{m}(x, \mu, \theta)
$$




\section{Conclusion}

In general, obtaining solutions of some integrodifferential equations are usually difficult. In our recent works we have used Walsh functions, Chebyshev polynomials and Lengendre polynomials in order to reduces these kind of equations. However our present work suggests that the Tau method can be a good approximation to the exact solutions. The application of the Tau method by using the orthogonal polynomials will be considered as a future work.

\section{Acknowledgments}

The authors thank the referee(s) for the helpful and significant comments that bring the attention of authors to the references [7-11] which update the bibliography. The authors gratefully also acknowledge that this research was partially supported by the University Putra Malaysia under the ScienceFund Grant no. 06-01-04-SF1050 and Research University Grant Scheme no. 05-01-09-0720RU.

\section{References}

[1] A. Haar, "Zur Theorie der orthogonalen Funktionensysteme," Mathematische Annalen, vol. 69, no. 3, pp. 331-371, 1910.

[2] P. Wojtaszczyk, A Mathematical Introduction to Wavelets, vol. 37 of London Mathematical Society Student Texts, Cambridge University Press, Cambridge, Mass, USA, 1997.

[3] R. E. A. C. Paley, "A remarkable series of orthogonal functions I," London Mathematical Society, vol. 34, pp. 241-264, 1932.

[4] R. E. A. C. Paley, "A remarkable series of orthogonal functions II," London Mathematical Society, vol. 34, pp. 265-279, 1932.

[5] N. J. Fine, "On the Walsh functions," Transactions of the American Mathematical Society, vol. 65, pp. 372-414, 1949.

[6] C. F. Chen and C. H. Hsiao, "A Walsh series direct method for solving variational problems," Journal of the Franklin Institute, vol. 300, no. 4, pp. 265-280, 1975.

[7] M. Asadzadeh, " $L_{2}$-error estimates for the discrete ordinates method for three-dimensional neutron transport," Transport Theory and Statistical Physics, vol. 17, no. 1, pp. 1-24, 1988.

[8] M. Asadzadeh, " $L_{p}$ and eigenvalue error estimates for the discrete ordinates method for twodimensional neutron transport," SIAM Journal on Numerical Analysis, vol. 26, no. 1, pp. 66-87, 1989.

[9] M. Asadzadeh, "A finite element method for the neutron transport equation in an infinite cylindrical domain," SIAM Journal on Numerical Analysis, vol. 35, no. 4, pp. 1299-1314, 1998.

[10] M. Asadzadeh and A. Kadem, "Chebyshev spectral- $S_{N}$ method for the neutron transport equation," Computers \& Mathematics with Applications, vol. 52, no. 3-4, pp. 509-524, 2006.

[11] M. Asadzadeh, P. Kumlin, and S. Larsson, "The discrete ordinates method for the neutron transport equation in an infinite cylindrical domain," Mathematical Models E Methods in Applied Sciences, vol. 2, no. 3, pp. 317-338, 1992.

[12] E. E. Lewis and W. F. Miller Jr., Computational Methods of Neutron Transport, John Wiley \& Sons, New York, NY, USA, 1984.

[13] M. Asadzadeh, "Analysis of a fully discrete scheme for neutron transport in two-dimensional geometry," SIAM Journal on Numerical Analysis, vol. 23, no. 3, pp. 543-561, 1986.

[14] A. V. Cardona and M. T. Vilhena, "A solution of linear transport equation using Walsh function and laplace transform," Annals of Nuclear Energy, vol. 21, pp. 495-505, 1994.

[15] M. S. Corrington, "Solution of differential and integral equations with walsh functions," IEEE Transactions on Circuit Theory, vol. 20, no. 5, pp. 470-476, 1973.

[16] E. L. Ortiz, "The tau method," SIAM Journal on Numerical Analysis, vol. 6, pp. 480-492, 1969.

[17] E. L. Ortiz and H. Samara, "An operational approach to the tau method for the numerical solution of nonlinear differential equations," Computing, vol. 27, no. 1, pp. 15-25, 1981. 


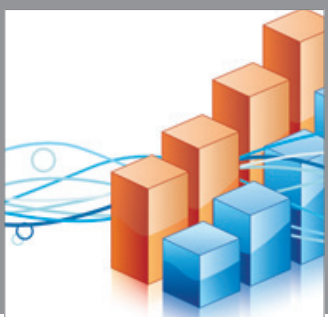

Advances in

Operations Research

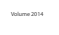

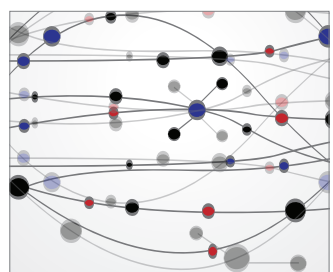

\section{The Scientific} World Journal
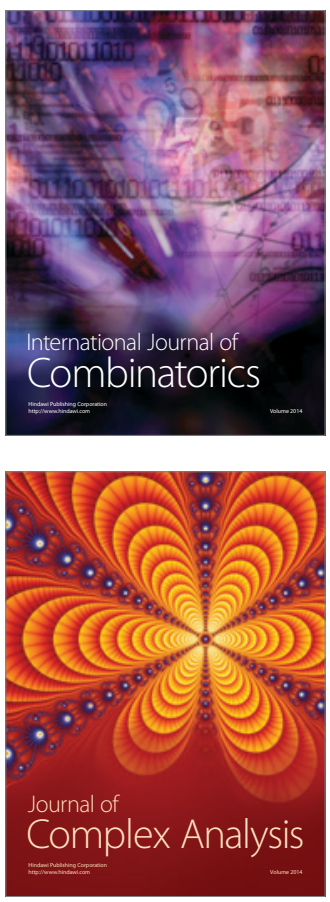

International Journal of

Mathematics and

Mathematical

Sciences
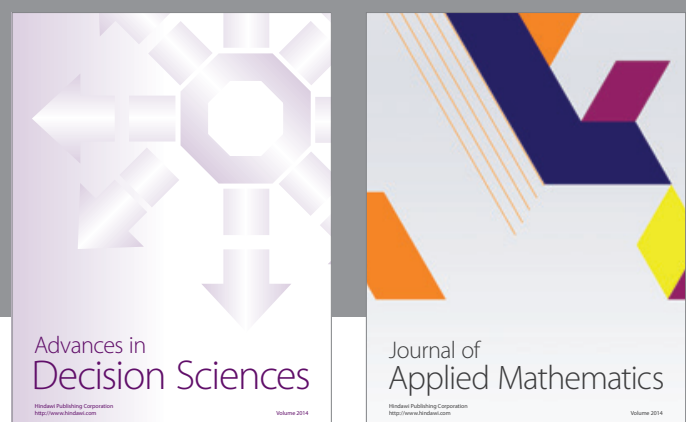

Journal of

Applied Mathematics
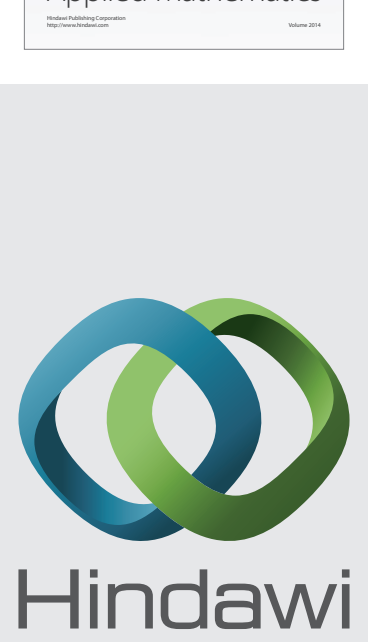

Submit your manuscripts at http://www.hindawi.com
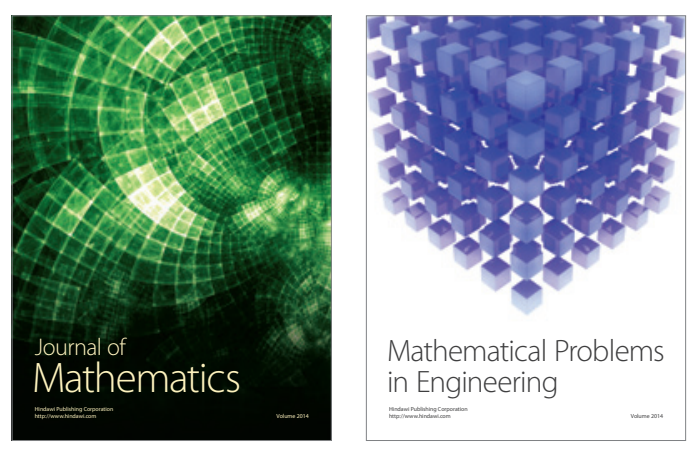

Mathematical Problems in Engineering
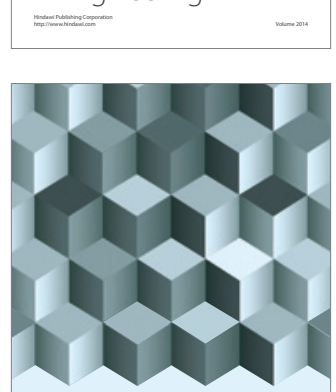

Journal of

Function Spaces
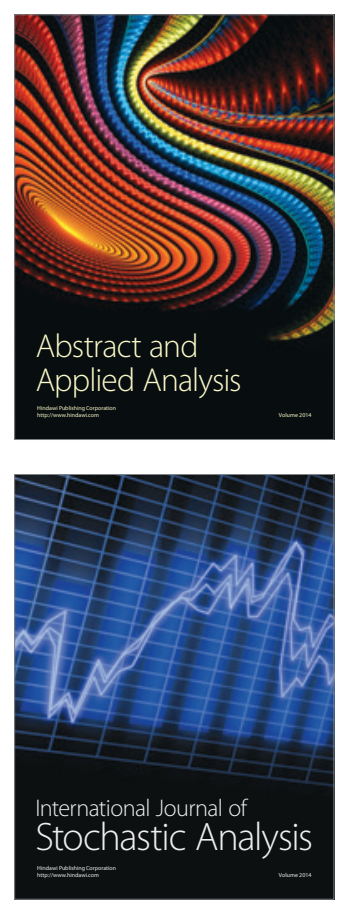

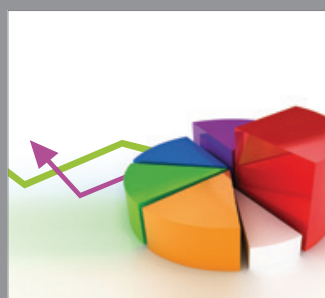

ournal of

Probability and Statistics

Promensencen
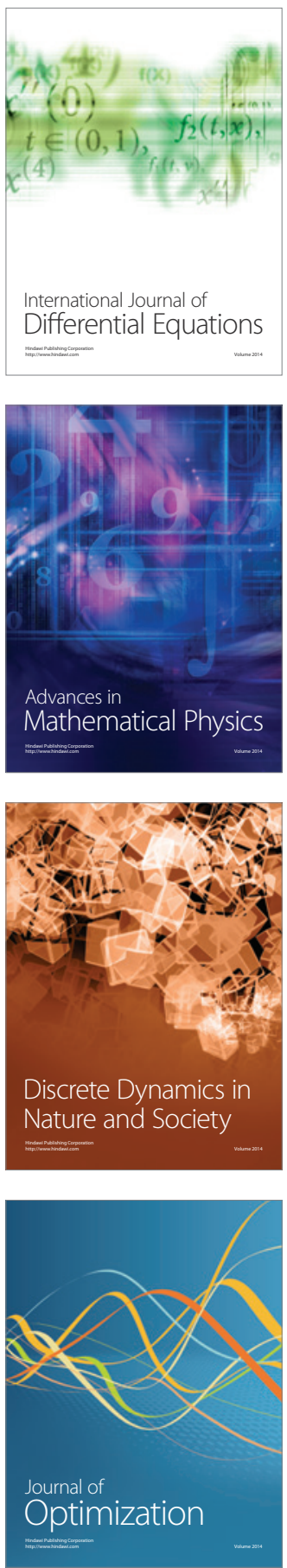